\title{
Voltage-Mediated Control of Spontaneous Bundle Oscillations in Saccular Hair Cells
}

\author{
Sebastiaan W. F. Meenderink, ${ }^{1}$ Patricia M. Quiñones, ${ }^{1}$ and Dolores Bozovic ${ }^{1,2}$ \\ ${ }^{1}$ Department of Physics and Astronomy, University of California, Los Angeles, California 90095, and ${ }^{2}$ California NanoSystems Institute, University of \\ California, Los Angeles, California 90095
}

Hair cells of the vertebrate vestibular and auditory systems convert mechanical inputs into electrical signals that are relayed to the brain. This transduction involves mechanically gated ion channels that open following the deflection of mechanoreceptive hair bundles that reside on top of these cells. The mechano-electrical transduction includes one or more active feedback mechanisms to keep the mechanically gated ion channels in their most sensitive operating range. Coupling between the gating of the mechanosensitive ion channels and this adaptation mechanism leads to the occurrence of spontaneous limit-cycle oscillations, which indeed have been observed in vitro in hair cells from the frog sacculus and the turtle basilar papilla. We obtained simultaneous optical and electrophysiological recordings from bullfrog saccular hair cells with such spontaneously oscillating hair bundles. The spontaneous bundle oscillations allowed us to characterize several properties of mechano-electrical transduction without artificial loading the hair bundle with a mechanical stimulus probe. We show that the membrane potential of the hair cell can modulate or fully suppress innate oscillations, thus controlling the dynamic state of the bundle. We further demonstrate that this control is exerted by affecting the internal calcium concentration, which sets the resting open probability of the mechanosensitive channels. The auditory and vestibular systems could use the membrane potential of hair cells, possibly controlled via efferent innervation, to tune the dynamic states of the cells.

Key words: frog; hair cell; sacculus; spontaneous oscillations

\section{Significance Statement}

The sensation of sound and balance starts by converting minute mechanical motions into electrical signals. This is accomplished by sensory hair cells, in which the opening and closing of mechanosensitive channels follows the motion of their hair bundles. These hair bundles can exhibit motility without an external drive. Underlying these spontaneous bundle oscillations are two coupled feedback mechanisms that improve the cell's response when mechanically stimulated. Here, we present synchronized optical and electrical recordings from cells with spontaneously oscillating bundles and show that the membrane potential may be a control parameter, tuning the cell's feedback processes. This control comes from modulation of the channel's open probability, which is affected by the concentration of calcium inside the cell.

\section{Introduction}

In vertebrates, the detection of seismic and acoustic signals involves specialized cells that are distributed across various endorgans within the ear (Hudspeth, 1989). These hair cells transduce mechanical vibrations into an electrical response. They derive their name from the mechanoreceptive organelle, the hair

Received April 14, 2015; revised Aug. 21, 2015; accepted Sept. 6, 2015.

Author contributions: S.W.F.M., P.M.Q., and D.B. designed research; S.W.F.M. and P.M.Q. performed research; S.W.F.M. analyzed data; S.W.F.M., P.M.Q., and D.B. wrote the paper.

This work was supported by National Institutes of Health Grant R01DC011380 to D.B.

The authors declare no competing financial interests.

Correspondence should be addressed to Dr. Dolores Bozovic, Department of Physics and Astronomy, University of

California, 1-129 Knudsen Hall, 475 Portola Plaza, Los Angeles, CA 90095. E-mail: bozovic@physics.ucla.edu.

DOI:10.1523/JNEUROSCI.1451-15.2015

Copyright $\odot 2015$ the authors $\quad 0270-6474 / 15 / 3514457-10 \$ 15.00 / 0$ bundle, which protrudes from their apical surface and consists of 20-300 cylindrical stereovilli arranged in rows. Within each bundle, the length of the stereovilli is graded, and the tallest row of stereovilli is often flanked by a kinocilium. Stereovilli of adjacent rows are connected by tip links. Transduction of mechanical stimuli into electrical output involves deflection of the hair bundle toward the tallest row, which pivots the stereovilli at their base and increases tension in the tip links that connect pairs of stereovilli. This opens mechano-sensitive transduction channels that are connected to the tip links and results in the influx of cations that depolarize the hair cell.

In addition to transducing mechanical deflections into electrical signals, hair cells may provide energy on a cycle-by-cycle basis to increase the excursions of the stimulus-induced vibrations (e.g., Hudspeth, 2008). Such an active mechanism would 
allow the hair bundles to overcome viscous damping by the inner-ear fluids. In the cochlea, amplification is thought to involve electromotility (prestin-mediated changes in the length of outer hair cells that depend on the membrane potential) (Zheng et al., 2000). In nonmammalian ears, prestin orthologs are expressed but show a reduction or absence of motor function (Tan et al., 2011); amplification has, however, been attributed to prestin in hair cells of the chicken basilar papilla (Beurg et al., 2013). The hair bundle could also provide mechanical amplification, either as an alternative to prestin (Crawford and Fettiplace, 1985; Chan and Hudspeth, 2005) or in conjunction with prestin (Kennedy et al., 2005). One hypothesis predicts that adaptation of the mechano-electrical transduction (MET) complex provides the energy for this active feedback (Vilfan and Duke, 2003). In frog saccular hair cells, adaptation involves intrastereovillar myosin motors and the much faster reclosure of the MET channels, with both processes dependent on the internal $\mathrm{Ca}^{2+}$ concentration (Hudspeth, 2008). The coupling between the gating of the MET channels and these adaption mechanisms has received considerable attention in both experimental (Martin et al., 2000) and theoretical studies. The active hair bundle has been proposed to follow dynamics of a system that is close to a Hopf (Choe et al., 1998; Camalet et al., 2000) or other (Ò Maoiléidigh et al., 2012) bifurcations. In the proximity of a critical point, the system exhibits high responsiveness to external stimuli, which could explain the sensitivity, dynamic range, and frequency selectivity of audition. This nonlinear system can cross the bifurcation into an unstable state, where it undergoes limit-cycle oscillations in the absence of input. Spontaneous bundle oscillations have been observed in hair cells of the turtle basilar papilla (Crawford and Fettiplace, 1985) and the frog sacculus (Martin and Hudspeth, 1999). They were shown to require an active mechanism (Martin and Hudspeth, 1999).

For this study, synchronized optical and electrophysiological recordings were obtained from bullfrog saccular hair cells that exhibited spontaneous hair bundle oscillations. We used a twocompartment chamber that separated the fluids contacting the apical and basolateral surfaces of the cells, creating an environment similar to that in vivo. These recordings were used to characterize the transduction process within the physiological range of deflections, without the need to mechanically load the hair bundle. In addition, the membrane potential is shown to control the dynamic state of the hair bundle, strongly modulating or suppressing innate motility. We show that this control is mediated through the internal calcium concentration, which presumably affects adaptation and thus modulates the MET channel open probability. By controlling the membrane potential of hair cells, possibly via efferent innervation, the auditory and vestibular systems may tune the cells to dynamic states where they show different degrees of sensitivity to external stimuli.

\section{Materials and Methods}

Biological preparation. Data presented in this manuscript were recorded from 41 hair cells from 29 adult North American bullfrogs (Rana catesbeiana) of either gender. Animals were anesthetized (pentobarbital: 150 $\mathrm{mg} / \mathrm{kg}$ ), double pithed, and decapitated following protocols approved by the University of California, Los Angeles Chancellor's Animals Research Committee (Protocol ARC 2006-043). The sacculus from either ear was dissected from the otic labyrinth in oxygenated, artificial perilymph solution (for a list of the used external solutions, see Table 1). The epithelium was then mounted in a two-compartment chamber, with the basolateral membranes of the hair cells exposed to artificial cesiumcontaining perilymph (i.e., perilymph in which $\sim 97 \%$ of the sodium was replaced by cesium) while their apical surfaces were bathed in artificial
Table 1. Ionic composition of external solutions (in $\mathrm{mm})^{a}$

\begin{tabular}{lccccc}
\hline & Perilymph & LCP & Cs $^{+}$-perilymph & Endolymph & NMDG- endolymph \\
\hline $\mathrm{Na}^{+}$ & 110 & 110 & 100 & 2 & 2 \\
$\mathrm{~K}^{+}$ & 2 & 2 & 2 & 118 & 3 \\
$\mathrm{Ca}^{2+}$ & 1.5 & 0.1 & 1.5 & 0.25 & 0.25 \\
$\mathrm{Cl}^{-}$ & 113 & 113 & 113 & 118 & 112 \\
$\mathrm{Na}^{+}$-pyruvate & 1 & 1 & 1 & - & - \\
$\mathrm{Creatine}^{+}$ & 1 & 1 & 1 & - & - \\
$\mathrm{Cs}^{+}$ & - & - & 10 & - & - \\
NMDG & - & - & - & - & 110 \\
\hline
\end{tabular}

${ }^{a}$ Each solution also contained 3 mm D-glucose and $5 \mathrm{~mm} \mathrm{HEPES}$, was adjusted to $\mathrm{pH} 7.3$ with $\mathrm{NaOH}$ (perilymph-based) or $\mathrm{KOH}$ (endolymph-based), and had an osmolality of $230 \mathrm{mmol} / \mathrm{kg}$.

endolymph. Following an 8 min enzymatic dissociation with $15 \mu \mathrm{g} / \mathrm{ml}$ Collagenase IV (Sigma-Aldrich), the otolithic membrane was removed from the epithelium. After confirming the presence of spontaneously oscillating hair bundles, the endolymph-like solution in the top compartment was replaced with artificial perilymph. This fluid exchange was achieved via gravity perfusion of the entire top compartment of the recording chamber. The same perfusion system was used to expose hair bundles to external solutions of various ionic compositions (Table 1). Unless stated otherwise, recordings from hair cells with spontaneously oscillating bundles were obtained with their apical surfaces exposed to low-calcium perilymph (LCP) containing $100 \mu \mathrm{M} \mathrm{Ca}{ }^{2+}$. During data acquisition, no solutions were perfused.

Imaging and tracking of hair bundle motility. The preparation was imaged in an upright optical microscope (Olympus BX51WI) with a waterimmersion objective (Olympus XLUMPlanFl, 20×, $0.95 \mathrm{NA}$ ). Images were further magnified (either $\sim 200 \times$ or $\sim 400 \times$ total magnification) and projected onto the sensor of a high-speed CMOS camera (Photron FASTCAM SA1.1; $117 \mathrm{~nm} /$ pixel resolution). We obtained videos at 1000 frames per second with an exposure time of $1 \mathrm{~ms}$. From these videos, the position of a hair bundle was determined with customized software (MATLAB 2011a, The MathWorks). Briefly, the position of the hair bundle in each movie frame was calculated as the center of gravity from its intensity profile along a row of pixels. To reduce the noise levels, this calculation was done for 11-15 adjacent rows of pixels, the results of which were averaged. The focal plane was at the tip of the hair bundle, and its position was derived for motions toward and away from the tallest row of stereovilli only. No angular corrections were applied to any of the recordings due to the relatively small size of the oscillations relative to the bundle height $(\sim 7 \mu \mathrm{m})$ (Hudspeth, 1989), and the absence of significant tilting in the preparation.

Electrophysiological recordings. In synchrony with video acquisition, we recorded currents under whole-cell voltage-clamp from hair cells using a patch-clamp amplifier (Molecular Devices Axopatch 200B) and a data acquisition system (National Instruments PXI-6251) that was under the control of software written in MATLAB (The MathWorks). Thin-walled borosilicate glass pipettes were pulled (Sutter Instruments P-97) to a resistance of 3-5 $\mathrm{m} \Omega$. Pipette tips were coated with beeswax (Fisher Scientific) to reduce their capacitance and were filled with a solution that contained the following (in $\mathrm{mM}$ ): $0.08 \mathrm{Ca}^{2+}, 111 \mathrm{Cl}^{-}, 5$ HEPES, 110 $\mathrm{Cs}^{+}, 10$ TEA, 1 EGTA, 2 ATP, 1 GTP, $3 \mathrm{Mg}^{+}$, pH 7.2, osmolality 210 $\mathrm{mmol} / \mathrm{kg}$. The free $\mathrm{Ca}^{2+}$ concentration of this solution was calculated to be $\sim 25$ nM (Max Chelator program; http://maxchelator.stanford.edu/ CaMgATPEGTA-TS.htm). Whole-cell configuration was achieved by breaking the hair cell's basolateral membrane after the resistance between the electrode and the cell exceeded $1 \mathrm{G} \Omega$. Placement of the recording pipette onto the cell membrane required a local interruption of the two-chamber configuration. Despite this, the ionic exchange between the two recording chambers seemed negligible over the time course of the recordings. The output of the patch-clamp amplifier was low-pass filtered at $10 \mathrm{kHz}$, digitized at $50 \mathrm{kHz}$, and stored for offline analysis. Series resistance $\left(R_{s}\right)$ ranged from 7 to $26 \mathrm{M} \Omega$ (mean: $17 \mathrm{M} \Omega$ ) and was not compensated for during the recordings, but was used to correct the reported membrane potentials $\left(V_{\mathrm{m}}\right)$ throughout the manuscript (see below). It was measured immediately before each recording by presenting a series of $5 \mathrm{mV}$, hyperpolarizing steps from a holding potential of -70 
$\mathrm{mV}$, and its value obtained from the amplifier's compensation dial. Electrode tip junction potentials were measured to be $<5 \mathrm{mV}$ and were not corrected. All experiments were performed at room temperature.

Data analysis. For the comparison between bundle position and transduction current, the latter was low-pass filtered (eighth-order Chebyshev Type I; cutoff frequency: $400 \mathrm{~Hz}$ ) and then downsampled to match the camera's frame rate. Next, slow trends in both recordings were estimated using local weighted regression (loess; second-order polynomial; bandwidth $30 \%$ of dataset; tri-cube weight function) and subtracted from the recorded signals. For each current recording, the slow loess trend line ( $\left.I_{\text {slow }}\right)$ was used to calculate $V_{\mathrm{m}}$, by correcting the command voltage $V_{\text {command }}(t)$ for the potential drop across the series resistance $\left(\mathrm{R}_{\mathrm{s}}\right)$ according to the following:

$$
V_{\mathrm{m}}(t)=V_{\text {command }}(t)-R_{\mathrm{s}} \times I_{\text {slow }}(t)
$$

To explore the effects of membrane potential on the spontaneous bundle oscillations, we obtained recordings from 11 hair cells, each one from a different frog. The cells were either clamped to a series of fixed membrane potentials, or their membrane potential was continuously varied in the depolarizing and hyperpolarizing direction. The rate of membranepotential change (typically $<20 \mathrm{mV} / \mathrm{s}$ ) was much smaller than the time scale of the spontaneous oscillation. The results from these two stimulus paradigms did not differ and are presented together. In each recording, the membrane potential was increased (depolarized) to at least $-10 \mathrm{mV}$, where all spontaneous bundle oscillations ceased.

Recordings were divided into segments that covered $10 \mathrm{mV}$ intervals in the membrane potential after correction for series resistance. This yielded 299 "equal- $V_{\mathrm{m}}$ " segments of paired current and displacement recordings; each of these segments was analyzed separately, as detailed below. First, the cross-correlation between the position and the MET current (normalized such that the auto-correlations at zero lag are identically 1.0 ) was calculated. If its maximum was $\geq 0.6$, the pair of recordings was included in the subsequent analysis $(n=243)$. Of the 56 equal- $V_{\mathrm{m}}$ segments thus excluded, 41 were at $V_{\mathrm{m}}>-25 \mathrm{mV}$, where the hair bundle no longer oscillated spontaneously. Next, the inverse cumulative distribution functions of the bundle position and the measured current were used to calculate the amplitude of the oscillation and the transduction current, defined to be the difference between the 975 and 25 permilles, respectively. To reduce the effects of noise in the subsequent analysis, equal- $V_{\mathrm{m}}$ segments for which the estimated transduction current $<50 \mathrm{pA}$ were also omitted from the dataset $(n=6)$. For the remaining 237 equal $-V_{\mathrm{m}}$ segments, we estimated the fraction of time that the bundle was deflected toward the tallest row of stereovilli and the mean frequency of the spontaneous oscillations. The former was calculated as the time during which the bundle's position was more positive than the average value of the 25 and 975 permilles, divided by the total time within the equal- $V_{\mathrm{m}}$ segment; the latter was calculated as the slope of a straight line that was fitted to the unwrapped, instantaneous phase of the bundle position. Here, the instantaneous phase was constructed based on the automatic detection of spontaneous bundle oscillations (Kao et al., 2013). Identical equal- $V_{\mathrm{m}}$ segments within one cell were analyzed separately, with the results presented as the mean.

To estimate the MET channel's gating force from these spontaneous bundle oscillations, we performed a fit of the instantaneous displacement-current data, for each equal- $V_{\mathrm{m}}$ segment, to a first-order Boltzmann function as follows:

$$
I(x)=I_{0}+\frac{I_{\max }}{\left(1+e^{\frac{-z\left(x-x_{0}\right)}{(k T)}}\right)}
$$

Here, $x$ represents hair bundle displacement, $x_{0}$ is the displacement at which the current is one-half of the maximum, and $z$ is the single-channel gating force. $I_{0}$ and $I_{\max }$ are factors to scale the open probability to the measured transduction current, and $k T$ is the energy, taken to be at room temperature $(T=298 \mathrm{~K})$. To minimize the effects of adaptation, only the steepest parts of the spontaneous bundle oscillations were considered in the fits. Data for motion toward and away from the tallest row of stereovilli were fitted independently in each equal- $V_{\mathrm{m}}$ segment.

The results from each fit were discarded if $<50$ data points were available, the goodness of fit did not exceed 0.65 , or if the open probability of the fitted function varied by $<0.65$ between the 25 and 975 permilles of the bundle excursions. The latter criterion was introduced to ensure that the sigmoidal shape of the Boltzmann function is reflected in the measured instantaneous current-displacement curve; it avoids the inclusion of equal- $V_{\mathrm{m}}$ segments in which the recorded currents do not show appreciable saturation over the range of observed bundle excursions. With these additional selection criteria, we obtained results for 115 equal- $V_{\mathrm{m}}$ segments for bundle motion toward the tallest row of stereovilli, and for 109 segments for motion in the opposite direction. Of these, 102 equal- $V_{\mathrm{m}}$ segments yielded estimates of the gating force for bundle motion in both directions. Only these were used to assess possible dependence of the gating force on the direction of bundle motion.

To study the effects of membrane potential on the position of the hair bundle, recordings were obtained by alternating the membrane potential of 31 hair cells ( $n=20$ animals) between a "holding potential" $(40,-10$, -30 , or $-70 \mathrm{mV}$ command voltage) and a range of different potentials ( -120 to $20 \mathrm{mV}$ in $20 \mathrm{mV}$ intervals) with a duty cycle of $50 \%$. Each membrane potential was held for $60 \mathrm{~ms}$, with the steps occurring in pseudorandom order, and presented at least 5 times. From these recordings, the hair bundle's steady-state position at each holding potential was calculated from the last $10 \mathrm{~ms}$ before each transition in membrane potential. To facilitate comparison across recordings, the steady-state bundle positions for each recording are presented such that a straight-line fit to the position versus membrane potential (for $V_{\mathrm{m}} \leq-40 \mathrm{mV}$ ) intersected the $x$-axis at $-120 \mathrm{mV}$. In addition to the bundle's steady-state position, we also quantified the size of a transient response, termed the flick (Cheung and Corey, 2006), which occurred immediately following a depolarization of the membrane potential. In the plots of the average signals, the membrane depolarization occurs either at the onset or the offset of the voltage step, depending on the holding potential used. The size of the flick was taken to be the difference between the mean bundle position in a $10 \mathrm{~ms}$ window immediately before the membrane depolarization and the minimum bundle position in a $5 \mathrm{~ms}$ window immediately following this step. Although the duration of the flick was often longer than $5 \mathrm{~ms}$, this window was sufficient to capture its absolute minimum in all of the recordings. The noise floor associated with the flick was calculated similarly, using the minimum of the mean bundle position within the same $10 \mathrm{~ms}$ time window that preceded the change in membrane potential.

All data analysis, including statistical analysis, used customized software that was developed in MATLAB (The MathWorks).

\section{Results}

\section{Transduction currents correlate with hair bundle position during spontaneous oscillations}

We obtained simultaneous optical and electrophysiological recordings from hair cells within the sensory epithelium of the American bullfrog ( $R$. catesbeiana) sacculus. The whole-cell patch-clamp method was used to control $V_{\mathrm{m}}$ and record receptor currents for individual hair cells. No effort was made to select for hair-cell location within the macula, nor for type of hair cell (Rutherford and Roberts, 2009). In synchrony with the current recordings, the hair bundle position was monitored using highspeed video techniques.

At negative membrane potentials, the inward currents increased following bundle deflections toward the tallest row of stereovilli, whereas bundle displacements in the opposite direction decreased these currents (Fig. 1A). This is consistent with prior measurements (Corey and Hudspeth, 1979), where bundle excursions, resulting from an imposed stimulus, were shown to open the MET channels and result in brief fluxes of cations. Apart from the full limit-cycle oscillations, hair bundles also showed smaller excursions from the baseline, which likewise resulted in 

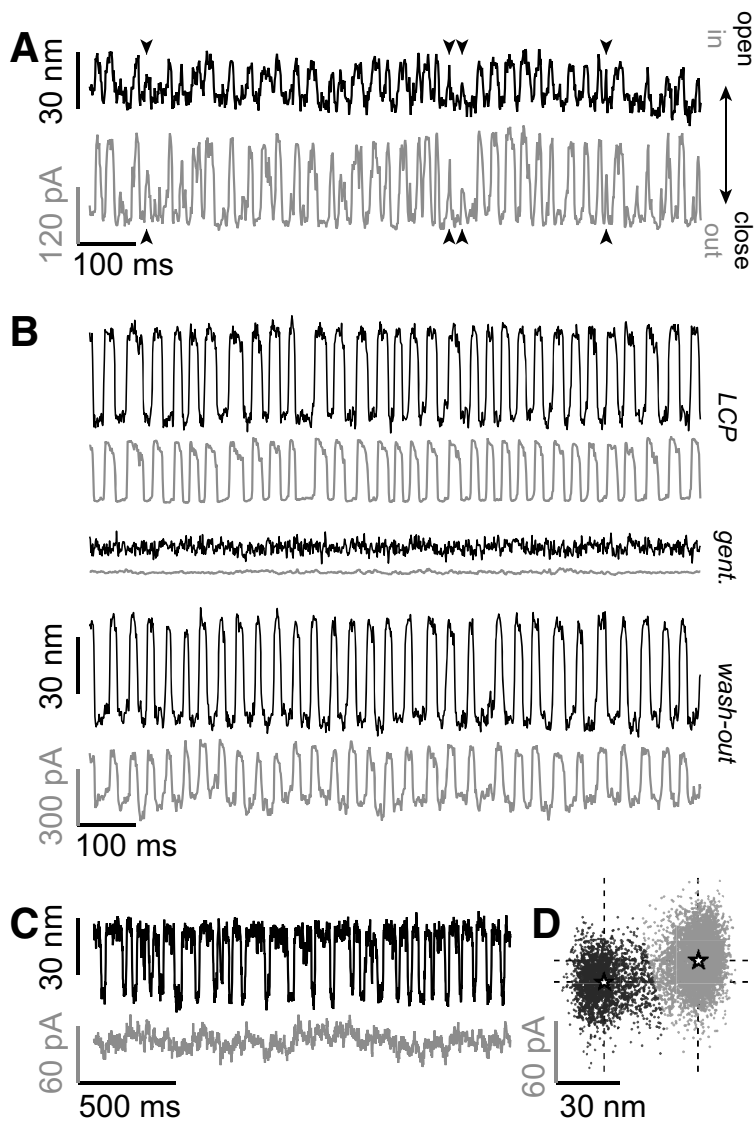

Figure 1. Transduction currents are correlated with spontaneous hair bundle oscillations. $A$, Bundle position (black) and MET current (gray) for a single hair cell that was held at a membrane potential of $-50 \mathrm{mV}$ in LCP solution. Upward deflections indicate hair bundle motion toward the tallest row of stereovilli (open) and an inward MET current (in), respectively. This differs from convention, where outward current is plotted as positive; it aids in observing the correlation between bundle position and MET current. The hair bundle oscillated spontaneously at 42.4 $\mathrm{Hz}$, and the two recordings were well correlated $(r=0.73)$. The hair bundle exhibited both full limit-cycle oscillations and motions of smaller amplitude (arrowheads) that likewise had a correlate in the MET current. $\boldsymbol{B}$, Bundle position (black) and MET current (gray) for a hair cell $\left(V_{\mathrm{m}}=-50 \mathrm{mV}\right)$ before, during, and after exposure of its apical surface to LCP containing 100 $\mu \mathrm{m}$ gentamicin. The presence of gentamicin, a blocker of the MET channels, abolishes both the spontaneous bundle oscillations and the measured currents. C, Bundle position (black) and MET current (gray) for a different hair cell that was held at a membrane potential of $-75 \mathrm{mV}$. The hair bundle was in artificial endolymph that had $\sim 97 \%$ of the monovalent cations replaced with N-methyl-o-glucamine (Table 1). The spontaneous oscillations (at $15.5 \mathrm{~Hz}$ ) did not have an obviously visible correlate in the recorded current $(r=-0.38)$. $\boldsymbol{D}$, Scatter plot of the bundle position versus the MET current for the recordings in $C$, partitioned in two clusters (different circles). Between these clusters, the mean inward current (white stars) was significantly larger when the bundle was deflected toward the tallest row of sterovilli (Welch's $t$ test, $\left.t_{(3718)}=-35.7, p<0.001\right)$.

smaller transduction currents (Fig. 1A, arrowheads). These smaller fluctuations likely reflect the stochastic nature of channel gating: only a fraction of the MET channels may open at any given time, leading to a smaller innate movement of the bundle.

To confirm that oscillations in the measured current result from modulation of the MET conductance, we exposed the apical surface of the hair cells to LCP (Table 1) containing $100 \mu \mathrm{M}$ gentamicin (Fig. $1 B$ ). This aminoglycoside is a known reversible blocker of the MET channel (Kroese et al., 1989). Its presence abolished the oscillations in the bundle position, as well as in the measured currents.

Figure $1 C$ shows that large transduction currents are not necessary for spontaneous bundle oscillations. Here, the apical sur- face of the epithelium was bathed in an endolymph-like solution in which $97 \%$ of the potassium was replaced by $N$-methyl-Dglucamine (a large monovalent cation that cannot pass through the MET channels; Table 1). Innate bundle oscillations persisted, at amplitudes comparable with, or larger than, those observed in LCP (compare Fig. $1 A, C$ ), whereas the large oscillations in the receptor current were eliminated. Nonetheless, bundle motion toward the tallest row of stereovilli did result in a small but significant change in the measured current (Fig. 1D), indicating that MET channels continued to open and close with the spontaneous bundle excursions. This measurement also confirms the functional integrity of the two-compartment configuration; if the presence of the patch electrode introduced a significant leak between the two compartments of the recording chamber, cations (primarily $\mathrm{Na}^{+}$) from the perilymph in the bottom chamber would infiltrate the upper compartment and lead to substantial inward currents.

\section{Membrane potential can modulate and suppress spontaneous hair bundle oscillations}

Typically, hair bundles from the frog sacculus do not oscillate spontaneously when the apical surface of the sensory epithelium is bathed in a solution that contains a high concentration of $\mathrm{Ca}^{2+}$ (e.g., artificial perilymph containing $1.5 \mathrm{mM} \mathrm{Ca}^{2+}$ ). Only when the bundles are exposed to artificial endolymph or perilymph with much reduced $\mathrm{Ca}^{2+}$ concentrations do they transition from the quiescent to the oscillatory regime. Most likely, the high external calcium concentration forces most of the MET channels to close, and thus prevent the occurrence of spontaneous bundle oscillations (Martin et al., 2003).

To manipulate the electrochemical driving force for calcium and test its effects on spontaneous bundle oscillations, we obtained recordings in which the membrane potential of individual hair cells was either clamped to a series of fixed potentials or slowly varied in a continuous fashion. Figure $2 \mathrm{~A}$ shows bundle position and MET currents for a hair cell that was held at various negative membrane potentials. The bundle position and the recorded current were again well correlated at all membrane potentials for which the bundle oscillated spontaneously, with movement toward the tallest row of stereovilli resulting in an inward transduction current. At sufficiently depolarized membrane potentials, the hair bundle oscillations were fully (and reversibly) suppressed, whereas at more hyperpolarized potentials, systematic changes could be observed in several characteristics of the innate bundle oscillations.

At $-90 \mathrm{mV}$, the hair bundle was mostly deflected toward the shortest row of stereovilli, and spontaneous oscillations briefly moved the bundle toward its tallest edge. This is also reflected in the measured currents, where the brief excursions toward the tallest row of stereovilli opened the MET channels and allowed an increase in the cationic influx. In contrast, at $-30 \mathrm{mV}$, the bundles remained primarily deflected toward the tallest row of stereovilli, and the spontaneous oscillations temporarily moved the bundle toward its shortest edge. These movements closed the transduction channels, as reflected in a reduced inward current. The transition from this mostly closed state (i.e., predominantly deflected toward the shortest row of stereovilli), observed at the most hyperpolarized membrane potentials, to the mostly open state, at the most depolarized membrane potentials, was gradual (Fig. 2B). The mean instantaneous frequency of bundle oscillation decreased with membrane potential (Fig. 2C), and no bundles showed any oscillations for membrane potentials $>-17$ $\mathrm{mV}$. In contrast, the amplitude of the bundle oscillations showed 
A

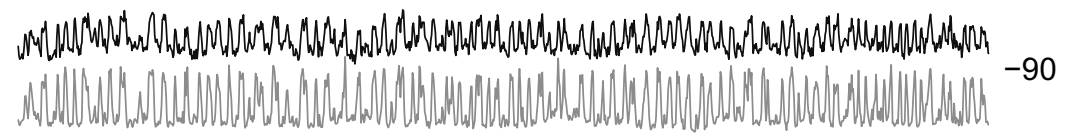

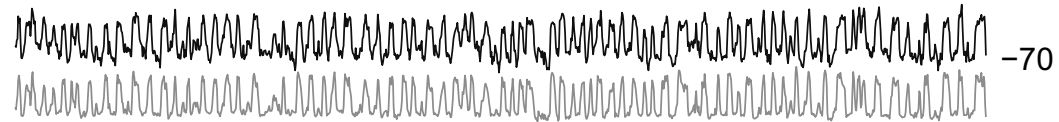
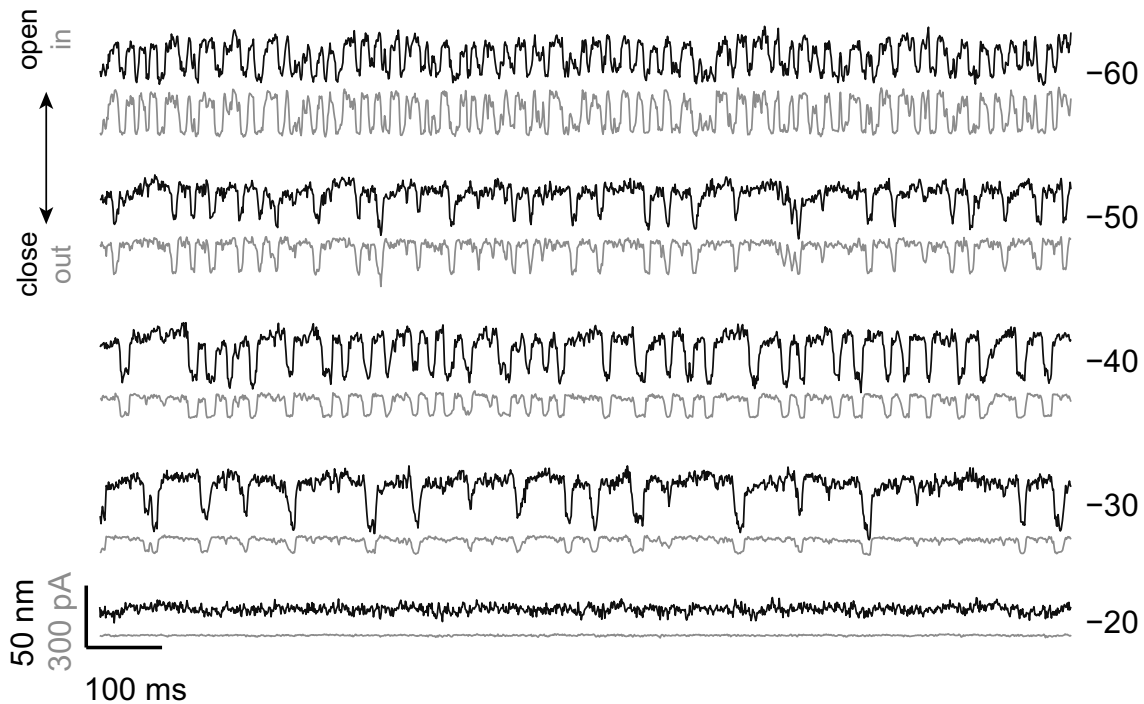

B

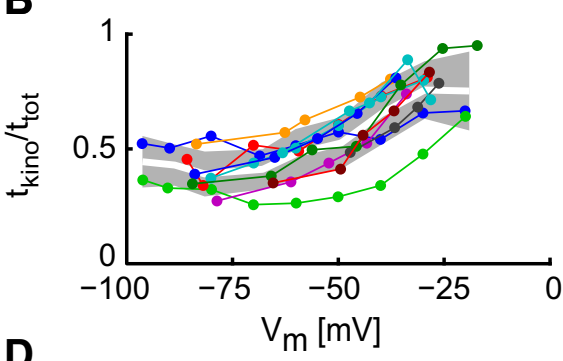

D
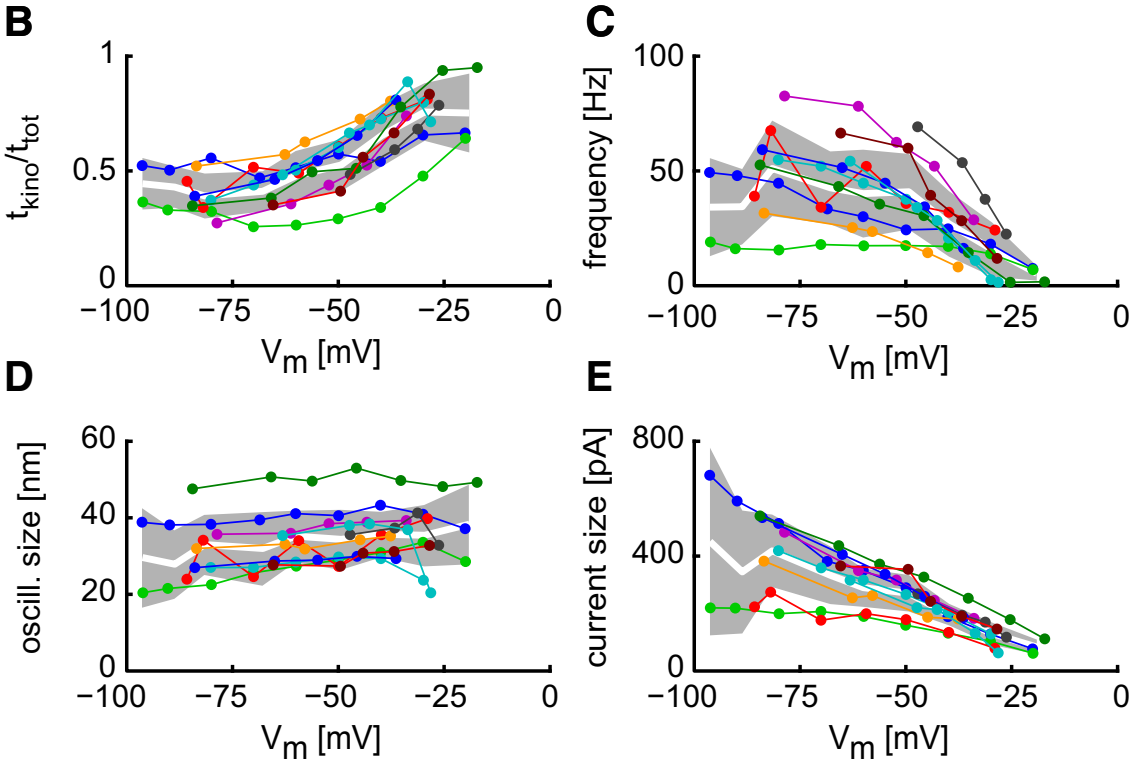

E

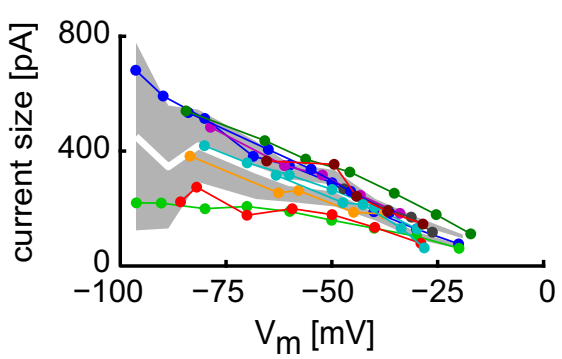

Figure 2. Membrane potential controls the occurrence of spontaneous hair bundle oscillations. $\boldsymbol{A}$, Series of recordings of bundle position (black) and MET current (gray) for a single hair cell that was held at different membrane potentials (command voltages, in $\mathrm{mV}$, are indicated on the right). Both bundle motion toward the tallest row of stereovilli (open) and the inward current (in) are positive. $\boldsymbol{B}-\boldsymbol{E}$, Various parameters as a function of $V_{\mathrm{m}}$ that were derived from recordings in 11 different hair cells. Each cell is represented by a series of circles, connected by lines. In all panels, each circle represents the mean result within an equal $V_{m}$ segment (see Materials and Methods). The white line and shaded gray area represent the mean and SD across cells, respectively. Data were omitted if the bundle did not oscillate spontaneously. $\boldsymbol{B}$, Fraction of time that the bundle is deflected toward the tallest row of sterovilli. $\boldsymbol{C}$, Mean instantaneous frequency of spontaneous bundle oscillations. $\boldsymbol{D}$, Amplitude of the bundle oscillation. $\boldsymbol{E}$, Magnitude of the transduction current. All recordings were obtained from cells with their hair bundles exposed to LCP solution. data yielded conductances for the MET complex between 2.0 and $7.8 \mathrm{nS}$ (median: $6.5 \mathrm{nS}$ ), and indicated a mean \pm SD reversal potential $(-2.7 \pm 11.1 \mathrm{mV}, n=11)$ that was not significantly different from zero $\left(t_{(10)}=-0.79 ; p=0.45\right)$, indicating that the channel is nonselective. We note that 2 of the 11 cells had conductances that were at least twice as small as the median value. No correlation between the measured MET conductance and the location of the hair cell within the epithelium, nor with size of the cell was observed. The variation in overall MET conductance could therefore reflect differences in the number of channels per hair bundle or signify the existence of multiple MET-channel types.

To estimate the MET-channel's gating force, both for motion toward and away from the tallest row of stereovilli, recordings like those presented in Figure $2 A$ were used to construct instantaneous currentdisplacement (I-X) curves. Spontaneous hair bundle oscillations are thought to arise from the interaction of two processes that act at different time scales: the gating of the MET channels, and (myosin-based) adaptation of the entire transduction complex. The hair bundle motion during a spontaneous oscillation reflects both of these processes: the fast transitions in the bundle position reflect channel gating, whereas the subsequent slower motion of the hair bundle is dominated by the effects of adaptation. To calculate the channels' instantaneous open probability from I-X curves, it is therefore necessary to use only those parts of the recordings that correspond to the gating of the channels, and to exclude the segments that also reflect bundle motion associated with adaptation. We included only those data that comprised the steep flanks of the bundle oscillations (Fig. 3A) to obtain the I-X relations for the gating-associated portions of bundle motility toward (Fig. $3 B$, red) and away from (Fig. 3B, blue) the tallest row of stereovilli, respectively. At each membrane potential, the two I-X relationships were fitted independently with a single Boltzmann function (Eq. 2), which represents a two-state model for the MET channel. This implicitly assumes that the I-X relation exhibits signs of current saturation over the extent of bundle displacements. Because spontaneous bundle oscillations need not result in complete a small, but significant, increase (mean slope $\pm \mathrm{SD}=0.1 \pm 0.04$ $\mathrm{nm} / \mathrm{mV} ; t_{(65)}=2.16, p=0.03$ ) with increasing membrane potential (Fig. 2D). Finally, the transduction currents were positive at negative membrane potentials and decreased in size with increasing membrane potential (Fig. 2E). Straight-line fits to the modulation of the MET-complex open probability (Martin et al., 2003), we used several selection criteria (see Materials and Methods) to analyze only those segments of the recordings for which a good fit to the Boltzmann function could be obtained. Figure $3 C$ shows that the analysis yields values for $\mathrm{I}_{\max }$ that are similar to the 
A

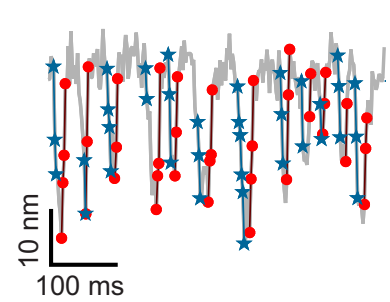

C

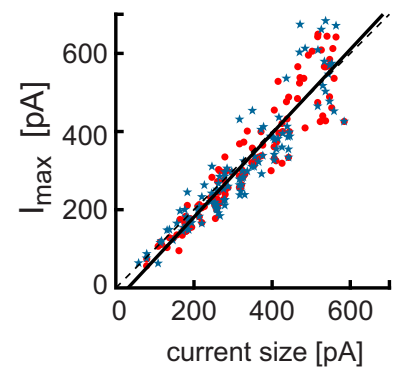

E

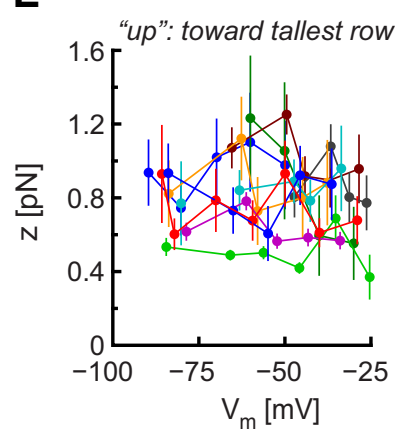

B

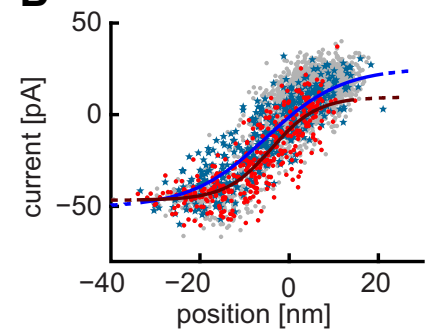

D

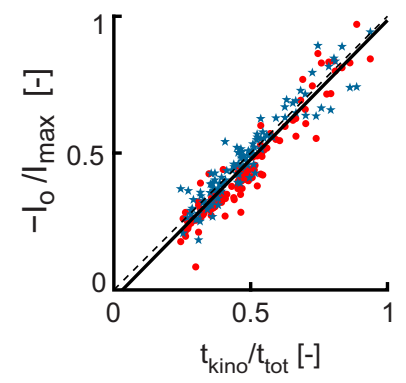

$\mathbf{F}$

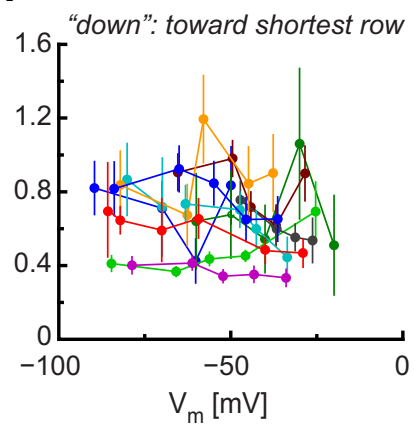

Figure 3. Gating force of the MET channels. $\boldsymbol{A}$, Hair bundle position for a hair cell that was clamped to a membrane potential of $-30 \mathrm{mV}$ (gray line). Motion toward the tallest row of stereovilli is positive. Those sections of the curve that corresponded to the initial, fast motions toward ("up"; red circles) or away from the tallest row of stereovilli ("down"; blue stars) were selected to be fit with a single Boltzmann function (see Eq. 2). B, Scatter plot of instantaneous current versus displacement for the recording in $\boldsymbol{A}$, including the fits that correspond to motions in the "up" (solid red line) and the "down" (solid blue line) directions. Dashed lines indicate extrapolations of the fits beyond the extent of bundle excursions. C, Comparison between I max $_{\text {man }}$ from the fit and the size of the transduction current (from Fig. 2E). Solid black line indicates the straight-line fit to these data $\left(y=1.07( \pm 0.06) x-32( \pm 0.06) ; r^{2}=0.86\right)$. Dashed line indicates equality. $\boldsymbol{D}$, Similar comparison to $\boldsymbol{C}$, obtained for the mean open probability of the MET complex $\left(y=1.02( \pm 0.05) x+0.03( \pm 0.02) ; r^{2}=0.89\right)$. Gating force $(z)$ for motion toward $(\boldsymbol{E})$ and away from $(\boldsymbol{F})$ the tallest row of stereovilli. Error bars indicate \pm 1 SD.

total MET current size (as given in Fig. 2E) obtained empirically. Also, estimates of the average MET-complex's open probability from Figure $2 B$ are almost identical to those calculated from the fit $\left(-\mathrm{I}_{\mathrm{o}} / \mathrm{I}_{\mathrm{max}}\right.$; Fig. $\left.3 D\right)$. These observations confirm that the Boltzmann function gives an appropriate description of the measured I-X relation.

Using the fits, we calculated the maximum modulation of the MET-channel's open probability. These $\Delta$ p $_{\text {open }}$ values were obtained by calculating the difference in the currents at the 25 and 975 permilles of the observed bundle excursions, normalized by $\mathrm{I}_{\max }$. For the cells included in the analysis, the mean $\Delta \mathrm{p}_{\text {open }}=$ 0.86 , where $94 \%$ (95 of 101) had a $\Delta \mathrm{p}_{\text {open }}>0.7$. Hence, bundle excursions of $<50 \mathrm{~nm}$ (Fig. $2 D$ ) are sufficient to modulate the MET-channel open probability over most of its dynamic range. This result provides additional evidence that the I-X curves are well described by the Boltzmann function.

Figure $3 E$ shows the single-channel gating force $(z)$ for motion toward the tallest row of stereovilli. It did not vary with the membrane potential $\left(t_{(49)}=0.66 ; p=0.51\right)$ and was on average 0.80 ( $\pm 0.20 \mathrm{SEM}) \mathrm{pN}(n=11$ cells $)$. Similarly, for bundle motion in the opposite direction (toward the shortest row of stereovilli; Fig. $3 F$ ), the membrane potential did not affect the gating force $\left(t_{(48)}=0.88 ; p=0.38\right)$; its mean $( \pm$ SEM) value was as follows: $0.66( \pm 0.17)$. These findings imply that the membrane potential does not strongly affect the stiffness of elastic elements that are in series with the transduction complex, at least not on the timescales probed in these experiments. A pairwise comparison between the gating forces for motion toward and away from the tallest row of stereovilli yielded $\mathrm{z}_{\text {toward }}-\mathrm{z}_{\text {away }}=0.13( \pm 0.34 \mathrm{SEM}) \mathrm{pN}$.

\section{Rapid changes in the membrane potential evoke bundle motility at two characteristic timescales}

A consequence of the voltage dependence of the MET-channel open probability would be that the resting position of the hair bundle varies with the membrane potential. To explore the voltage-mediated changes in bundle position, recordings were obtained from hair cells for which the bundles were immersed in perilymph, and the membrane potential was varied using a series of steps. Two responses were observed: a change in the steadystate position and a transient response (Fig. 4). Both types of bundle responses had a correlate in the simultaneously recorded receptor currents (Fig. 4A). These currents are presumably carried by both the MET complex and voltage-gated calcium channels in the basolateral membrane and are not further discussed.

To determine whether the two responses in the bundle's position varied with membrane potential or with the size of the potential change, recordings were obtained from different holding potentials (Fig. 4A). The hair bundle steady-state position depended on the membrane potential in a nonlinear fashion (Fig. $4 B, D)$. At membrane potentials $<-40 \mathrm{mV}$, the bundle was deflected toward the shortest row of stereovilli and there was little variation in the bundle position at different potentials. For membrane potentials $>-40 \mathrm{mV}$, the steady-state position shifted such that the hair bundle was deflected more toward its row of tallest stereovilli at more depolarized potentials. This shift in the steady-state deflection toward the kinocilium reached a maximum $\sim 0 \mathrm{mV}$; for more depolarized potentials, the hair bundle's resting position was again poised toward the row of shortest stereovilli.

The transient response of the hair bundle depended on the change in the membrane potential, rather than on the potential itself (Fig. 4C,E). These rapid movements occurred following membrane depolarizations $>50 \mathrm{mV}$, and their size linearly increased with the size of the depolarizing step. Transient responses were occasionally observed for hyperpolarizing voltage steps but were always of much smaller size and limited to relatively hyperpolarized potentials; they are not further discussed here. A similar response has been studied in hair cells from other species (Ricci et al., 2000). Following Cheung and Corey (2006), we will refer to this transient response as the "flick."

To further test whether the membrane-potential-induced changes in the bundle position were driven by calcium influx into the hair bundle, recordings were obtained from hair bundles that were successively exposed to perilymph $\left(1.5 \mathrm{~mm}\left[\mathrm{Ca}^{2+}\right]\right)$ and $\operatorname{LCP}\left(100 \mu \mathrm{M}\left[\mathrm{Ca}^{2+}\right]\right)$ solutions (Fig. $5 A$ and $B$, respectively). The 15 -fold difference in the external calcium concentration between these solutions affected both the steady-state position of the hair bundle (Fig. 5C) and the size of the flick (Fig. 5D). The former can 
A
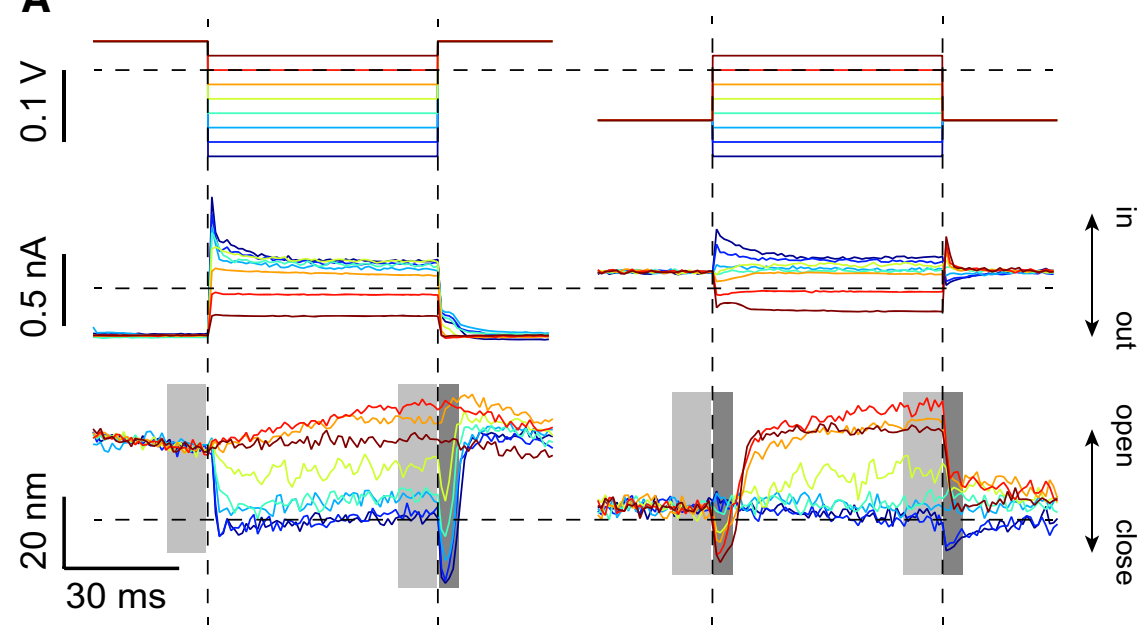

B
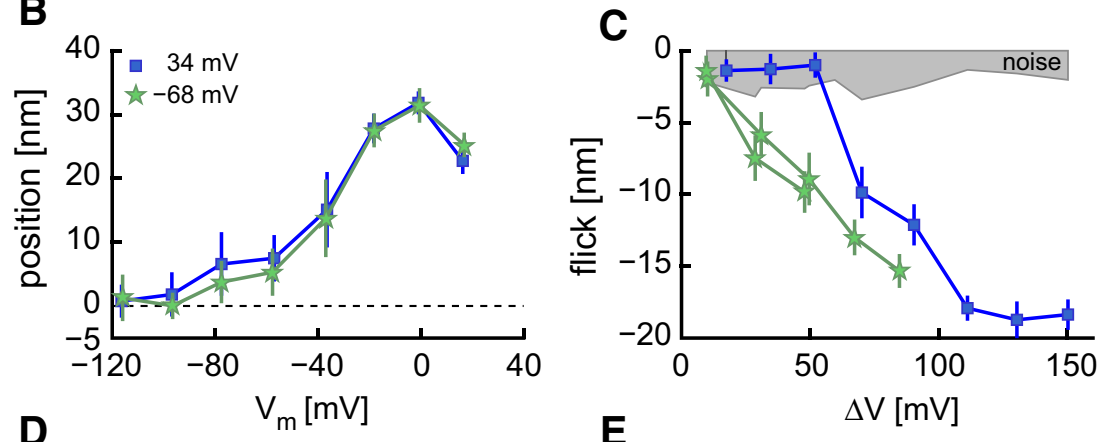

E
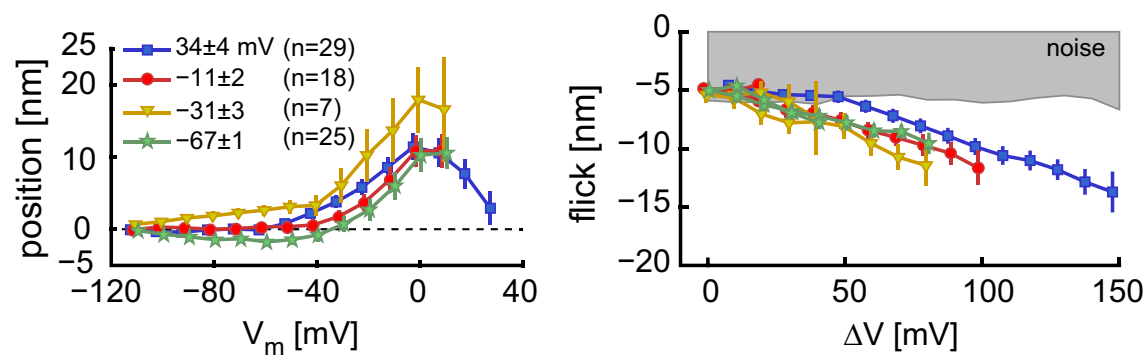

\section{Discussion}

Properties of the transduction complex In this manuscript, we presented recordings of hair bundle position and receptor current, obtained from saccular hair cells. The hair bundles of these cells oscillated spontaneously, over some range of membrane potentials. The oscillations allowed us to study MET without the need to mechanically load the hair bundles. We found that the bundle position and transduction currents were strongly correlated, not only during the main limit cycle oscillations (Martin et al., 2003), but also for smaller excursions. These were frequently observed from either the channel-open or the channel-closed position, and show that even minor changes in the bundle position lead to the opening/closing of a fraction of the transduction channels. As expected (e.g., Corey and Hudspeth, 1979), excursions toward the tallest row of stereovilli were excitatory, allowing the flux of cations, whereas motions in the opposite direction closed the channels.

The amplitude of the full limit cycle oscillation sufficed to open or close a large fraction of the MET channels, indicating an operating range for bundle deflections of $<50 \mathrm{~nm}$. This narrow operating range was also reflected by the calculated gating forces (Fig. 3). Their relatively large values (between 350 and $1250 \mathrm{fN}$ ) (see also Howard and Hudspeth, 1988; van Netten and Kros, 2000), as well as the correlation between the MET currents and bundle position down to the smallest excursions (Fig. $1 A$, arrowheads), indicate a high sensitivity of the transduction apparatus to mechanical motion. The operating range we observe is narrower than that obtained in prior studies in which the bundles were mechanically deflected to obtain I-X curves (e.g., Eatock et al., 1987; Cheung and Corey, 2006; Johnson et al., 2011).

Sharp and substantial changes in the membrane potential elicited sudden bundle movements (Figs. 4, 5) lasting a few milliseconds, which were only weakly af-

be explained by the change in the electrochemical gradient for calcium, which results in a $34.8 \mathrm{mV}$ shift in the Nernst potential. That is, differences between the steady-state position versus $V_{\mathrm{m}}$ curves were minimized when data obtained in LCP were compared with the responses in perilymph measured at membrane potentials that were $34.5 \mathrm{mV}$ (interquartile range: $24.6-40.1 \mathrm{mV}$, $n=18$ ) more depolarized (Fig. $5 E, F$ ).

The size of the flick slightly increased as a result of the large decrease in external calcium concentration (median: $2.16 \mathrm{~nm}$; Wilcoxon signed rank test: $Z=-7.29 ; p<0.001$; Fig. $5 D, G$ ). This was, however, only observed in a subset of the recordings and did not correlate with the expected change in the Nernst potential. The changed calcium influx may exert a small and transient effect on the stiffness of the hair bundle. fected by the external calcium concentration. The size of the transient response was independent of the membrane potential (the magnitude of the applied voltage step determined its amplitude) and occurred for relatively large $(>40 \mathrm{mV})$ depolarizations. A similar response has been observed in hair cells of the turtle papilla (Ricci et al., 2000) and in isolated hair cells from the bullfrog sacculus (Cheung and Corey, 2006), and was referred to as the "flick." In contrast to our findings, the responses measured in those experiments were of smaller amplitude and were symmetric with respect to the polarity of the applied voltage steps. The flick most likely results from a voltage-sensing element that is part of the transduction complex, but any functional role for this transient response is unknown. We note that these transient ex- 

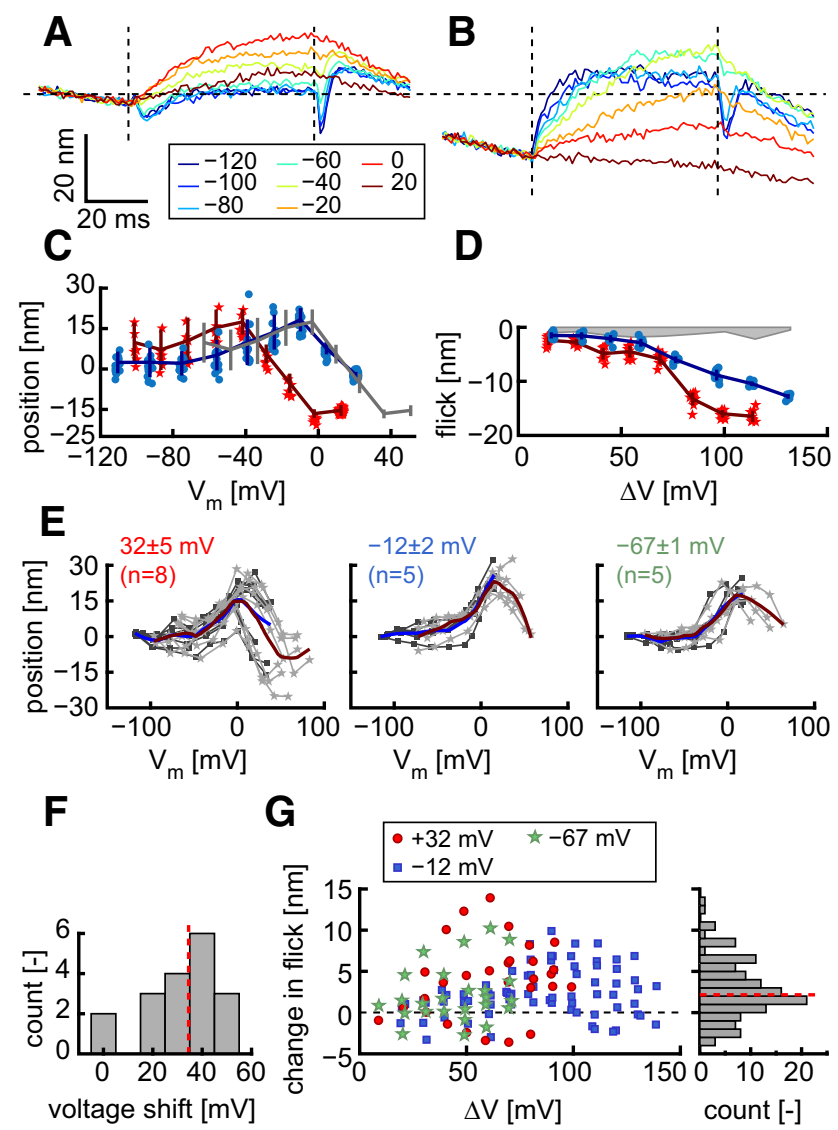

Figure 5. Effect of the membrane potential on hair bundle position is calcium-mediated. $\boldsymbol{A}$, Position of a hair bundle exposed to perilymph in response to a series of steps in the membrane potential (from $20 \mathrm{mV}$ ). See legend (in $\mathrm{mV}$ ) for the step command voltages. $\boldsymbol{B}$, Similar recording from the same hair bundle as in $\boldsymbol{A}$, exposed to LCP solution. Because of increased currents, voltage steps are from $12 \mathrm{mV}$. Vertical dashed lines indicate the onset and offset of the steps. Horizontal dashed line indicates zero bundle position from $\boldsymbol{C}$. Scale bars apply to $\boldsymbol{A}, \boldsymbol{B}$. C, Mean ( $\pm S D$ ) hair bundle position and individual responses in perilymph (red stars), and LCP solutions (blue circles), for the recordings in $\boldsymbol{A}, \boldsymbol{B}$. Gray curve represents the data obtained in LCP solution but shifted horizontally to maximally overlap with the data obtained in perilymph (shift $=38.4$ $\mathrm{mV})$. $\boldsymbol{D}$, Size of the flick for the recordings in $\boldsymbol{A}, \boldsymbol{B}$, same symbols as in $\boldsymbol{C}$. Shaded gray area represents the noise floor for the estimate of the flick size. $\boldsymbol{E}$, Mean bundle positions in perilymph (dark gray squares) and horizontally shifted mean bundle positions in LCP (light gray stars) for recordings stepped from different membrane potentials. Blue and red lines indicate loess trend lines (second-order polynomial; bandwidth: $35 \%$ of data; tri-cube weight function) for the perilymph and LCP data, respectively. $\boldsymbol{F}$, Histogram (10 $\mathrm{mV}$ bins) of the applied horizontal shifts in $\boldsymbol{E}$ to maximally overlap the bundle positions. The median shift of $34.5 \mathrm{mV}$ (dashed red line; interquartile range: 24.6 to $40.1 \mathrm{mV}$ ) matched the change in the Nernst potential for calcium $(34.8 \mathrm{mV}$ ) between the two solutions. $\mathbf{G}$, Left, Pairwise comparison between the flick size in perilymph and LCP. Positive values indicate that the flick is larger in LCP. Right, Histogram ( $1 \mathrm{~nm}$ bins) of these data, which had a median value of $2.7 \mathrm{~nm}$ (red dashed line) that was different from zero (Wilcoxon signed rank; $Z=-7.29, p<0.001$ ).

cursions led to bundle movements that extended beyond the steady-state positions reached at the smallest (i.e., most hyperpolarized) potentials.

\section{Control of calcium entry and its effects}

At hyperpolarized potentials $(<-40 \mathrm{mV}$; in perilymph), the hair bundles were deflected toward the shortest rows of stereovilli (Figs. 2B, 4C). This result is consistent with predictions based on fast adaptation (Bozovic and Hudspeth, 2003; Martin et al., 2003; for review, see LeMasurier and Gillespie, 2005): an increase in the electrostatic drive for calcium entry into the cell leads to closure of the transduction channels. For increas- ingly depolarized membrane potentials, the driving force for calcium entry is reduced, lowering the internal calcium concentration and thus increasing the channel open probability. As a consequence, the bundles were increasingly deflected toward their tallest edge, as the cell was depolarized. By controlling calcium entry, the membrane potential therefore modulates the channel open probability and hence affects the average position of the hair bundle.

In addition to its effects on fast adaptation, calcium also affects slow, myosin-based adaptation (Eatock et al., 1987; Assad et al., 1989; Hudspeth, 1989; Wu et al., 1999). At depolarized potentials $(\geq 0 \mathrm{mV})$, the channels are increasingly in the open state, but the calcium influx is decreased, and hence the rate of slippage of the myosin-motors along the actin filaments is reduced. As a result, the continuous climbing of the motors increases the tension in the tip links, gradually deflecting the bundle back toward its shortest edge. The combined effect of calcium on fast and slow adaptation leads to the observed nonmonotonic dependence of the steady-state position on membrane potential (Fig. 4D).

Although calcium is known to modulate slow adaptation by affecting the myosin motor slippage rate, its site of action for fast adaptation is unknown. Calcium affects the channel open probability at rest, but whether this modulation occurs directly at the channel (Cheung and Corey, 2006) or via some closely associated viscoelastic element (Bozovic and Hudspeth, 2003; Roongthumskul et al., 2011) is not known. A recent study of mammalian outer hair cells even raised the possibility of calcium-independent fast adaptation (Peng et al., 2013); however, different results were obtained under different stimulus protocols (Corns et al., 2014). Our findings indicate calcium-dependent fast adaptation, reflected in the strong impact of electrostatic potential on the channels' open probability (Fig. 2B). However, the measured gating force did not vary with the membrane potential, suggesting that there was no strong modulation of the stiffness of elastic elements in series with the MET complex in response to variation of the rate of calcium entry. We note that such modulation may occur at timescales different from those probed with the presented experiments.

\section{Active process and its modulation}

The dynamics of the hair bundle have been modeled using systems of coupled nonlinear differential equations (Choe et al., 1998, Eguíluz et al., 2000; Camalet et al., 2000). Such models predict the existence of different regimes, exhibiting the quiescent state or spontaneous limit cycle oscillation, separated by critical points. A control parameter determines in which regime the dynamic system resides, and can be used to tune the system near a critical point. In the proximity of a critical point, the models exhibit high responsiveness to external stimuli. Depending on the complexity of the model, the transition can occur via different types of bifurcations (Han and Neiman, 2010; Ó Maoiléidigh et al., 2012), which determine the exact response near the critical point.

The biological nature of the control parameter has not been established, although several parameters that affect the dynamic state of the hair bundle have been identified. These include the external calcium concentration (Martin et al., 2003), mechanical loading (Ó Maoiléidigh and Hudspeth, 2013), and the steady-state deflection of the bundle (Fredrickson-Hemsing et al., 2012). These parameters, however, are inextricably linked, and a change in any of them will 
affect the others. For example, a mechanical offset of the hair bundle changes the channel open probability, and hence the influx of calcium into the cell. The same change in $\mathrm{Ca}^{2+}$ influx can be accomplished by variation of the electrochemical gradient across the membrane (i.e., changing the external calcium concentration or the membrane potential).

Spontaneous oscillations of the hair bundle have been observed in vitro (Crawford and Fettiplace, 1985; Benser et al., 1996) and shown to require an energy-consuming mechanism (Martin et al., 2001). It was demonstrated that these large, noisy oscillations can be readily entrained by a much weaker drive, leading to a significant amplification of the response (Martin and Hudspeth, 1999). The absence of spontaneous oscillation, however, need not indicate that the system is passive; the same set of nonlinear equations describes the behavior in both the quiescent and oscillatory regime, with the differences captured by different values of the control parameters. The spontaneous motility arises in preparations from which the otolithic membrane has been removed, and hence under conditions in which the natural loading and coupling of the bundles has been changed. In the bullfrog sacculus, the presence of this overlying membrane was shown to suppress the innate motility (Strimbu et al., 2010). A hair bundle that is mechanically loaded so as to be poised in the quiescent regime can exhibit sporadic spike-like excursions (Roongthumskul and Bozovic, 2015). These spikes can be entrained by weak signals and provide significant enhancement of the bundle's response to applied stimuli. Whether spontaneous oscillations can arise in vivo remains unknown; however, either the oscillatory or the quiescent regime can exhibit an active response.

We showed that the membrane potential can tune the hair bundle toward or away from the regime of spontaneous oscillation and thus affect its dynamic state. Our data indicate that the membrane potential exerts its influence on bundle mechanics by affecting the internal calcium concentration in the cell. In contrast to variation of the external calcium concentration (Martin et al., 2003) or the mechanical loading of the hair bundle (Fredrickson-Hemsing et al., 2012), which are either constant or can only be modulated very slowly, the membrane potential can be changed on a much faster timescale. By controlling the hair-cell membrane potential, possibly via the efferent system, the auditory and vestibular sensory organs may thus be constantly self-tuning to a dynamic state where they show high sensitivity to external stimuli.

\section{References}

Assad JA, Hacohen N, Corey DP (1989) Voltage dependence of adaptation and active bundle movement of bullfrog saccular hair cells. Proc Natl Acad Soc U S A 86:2918-2922. CrossRef Medline

Benser ME, Marquis RE, Hudspeth AJ (1996) Rapid, active hair bundle movements in hair cells from the bullfrog's sacculus. J Neurosci 16:56295643. Medline

Beurg M, Tan X, Fettiplace R (2013) A prestin motor in chicken auditory hair cells: active force generation in a nonmammalian species. Neuron 79:69-81. CrossRef Medline

Bozovic D, Hudspeth AJ (2003) Hair-bundle movements elicited by transepithelial electrical stimulation of hair cells in the sacculus of the bullfrog. Proc Natl Acad Soc U S A 100:958-963. CrossRef Medline

Camalet S, Duke T, Jülicher F, Prost J (2000) Auditory sensitivity provided by self-tuned critical oscillations of hair cells. Proc Natl Acad Soc U S A 97:3183-3188. CrossRef Medline

Chan DK, Hudspeth AJ (2005) $\mathrm{Ca}^{2+}$ current-driven nonlinear amplification by the mammalian cochlea in vitro. Nat Neurosci 8:149-155. CrossRef Medline
Cheung EL, Corey DP (2006) $\mathrm{Ca}^{2+}$ changes the force sensitivity of the haircell transduction channel. Biophys J 90:124-139. CrossRef Medline

Choe Y, Magnasco MO, Hudspeth AJ (1998) A model for amplification of hair-bundle motion by cyclical binding of $\mathrm{Ca}^{2+}$ to mechanoelectricaltransduction channels. Proc Natl Acad Soc U S A 95:15321-15326. CrossRef Medline

Corey DP, Hudspeth AJ (1979) Ionic basis of the receptor potential in a vertebrate hair cell. Nature 281:675-677. CrossRef Medline

Corns LF, Johnson SL, Kros CJ, Marcotti W (2014) Calcium entry into stereocilia drives adaptation of the mechanoelectrical transducer current of mammalian cochlear hair cells. Proc Natl Acad Soc U S A 111:1491814923. CrossRef Medline

Crawford AC, Fettiplace R (1985) The mechanical properties of ciliary bundles of turtle cochlear hair cells. J Physiol 364:359-379. CrossRef Medline

Eatock RA, Corey DP, Hudspeth AJ (1987) Adaptation of mechanoelectrial transduction in hair cells of the bullfrog's sacculus. J Neurosci 7:28212836. Medline

Eguíluz VM, Ospeck M, Choe Y, Hudspeth AJ, Magnasco MO (2000) Essential nonlinearities in hearing. Phys Rev Lett 84:5232-5235. CrossRef Medline

Fredrickson-Hemsing L, Strimbu CE, Roongthumskul Y, Bozovic D (2012) Dynamics of freely oscillating and coupled hair cell bundles under mechanical deflection. Biophys J 102:1785-1792. CrossRef Medline

Han L, Neiman AB (2010) Spontaneous oscillations, signal amplification, and synchronization in a model of active hair bundle mechanics. Phys Rev E 81:041913. CrossRef Medline

Howard J, Hudspeth AJ (1988) Compliance of the hair bundle associated with gating of mechanoelectrical transduction channels in the bullfrog's saccular hair cell. Neuron 1:189-199. CrossRef Medline

Hudspeth AJ (1989) How the ear's works work. Nature 341:397-404. CrossRef Medline

Hudspeth AJ (2008) Making an effort to listen: mechanical amplification in the ear. Neuron 59:530-545. CrossRef Medline

Johnson SL, Beurg M, Marcotti W, Fettiplace R (2011) Prestin-driven cochlear amplification is not limited by the outer hair cell membrane constant. Neuron 70:1143-1154. CrossRef Medline

Kao A, Meenderink SW, Bozovic D (2013) Mechanical overstimulation of hair bundles: suppression and recovery of active motility. PLoS One 8:e58143. CrossRef Medline

Kennedy HJ, Crawford AC, Fettiplace R (2005) Force generation by mammalian hair bundles supports a role in cochlear amplification. Nature 433:880-883. CrossRef Medline

Kroese AB, Das A, Hudspeth AJ (1989) Blockage of the transduction channels of hair cells in the bullfrog's sacculus by aminoglycoside antibiotics. Hear Res 37:203-217. CrossRef Medline

LeMasurier M, Gillespie PG (2005) Hair-cell mechanotransduction and cochlear amplification. Neuron 48:403-415. CrossRef Medline

Martin P, Hudspeth AJ (1999) Active hair-bundle movements can amplify a hair cell's response to oscillatory mechanical stimuli. Proc Natl Acad Soc U S A 96:14306-14311. CrossRef Medline

Martin P, Mehta AD, Hudspeth AJ (2000) Negative hair-bundle stiffness betrays a mechanism for mechanical amplification by the hair cell. Proc Natl Acad Soc U S A 97:12026-12031. CrossRef Medline

Martin P, Hudspeth AJ, Jülicher F (2001) Active hair-bundle movements can amplify a hair cell's response to oscillatory mechanical stimuli. Proc Natl Acad Soc U S A 98:14380-14385. CrossRef Medline

Martin P, Bozovic D, Choe Y, Hudspeth AJ (2003) (2003) Spontaneous oscillation by hair bundles of the bullfrog's sacculus. J Neurosci 23:45334548. Medline

Ó Maoiléidigh D, Hudspeth AJ (2013) Effects of cochlear loading on the motility of active outer hair cells. Proc Natl Acad Soc U S A 110:54745479. CrossRef Medline

Ó Maoiléidigh D, Nicola EM, Hudspeth AJ (2012) The diverse effects of mechanical loading on active hair cells. Proc Natl Acad Soc U S A 109: 1943-1948. CrossRef Medline

Peng AW, Effertz T, Ricci AJ (2013) Adaptation of mammalian auditory hair cell mechanotransduction is independent of calcium entry. Neuron 80:960-972. CrossRef Medline

Ricci AJ, Crawford AC, Fettiplace R (2000) Active hair bundle motion linked to fast transducer adaptation in auditory hair cells. J Neurosci 20:7131-7142. Medline 
Roongthumskul Y, Bozovic D (2015) Mechanical amplification exhibited by quiescent saccular hair bundles. Biophys J 108:53-61. CrossRef Medline

Roongthumskul Y, Fredrickson-Hemsing L, Kao A, Bozovic D (2011) Multiple-timescale dynamics underlying spontaneous oscillations of saccular hair bundles. Biophys J 101:603-610. CrossRef Medline

Rutherford MA, Roberts WM (2009) Spikes and membrane potential oscillations in hair cells generate periodic afferent activity in the frog sacculus. J Neurosci 29:10025-10037. CrossRef Medline

Strimbu CE, Kao A, Tokuda J, Ramunno-Johnson D, Bozovic D (2010) Dynamic state and evoked motility in coupled hair bundles of the bullfrog sacculus. Hear Res 265:38-45. CrossRef Medline

Tan X, Pecka JL, Tang J, Okoruwa OE, Zhang Q, Beisel KW, He DZ (2011) From zebrafish to mammal: functional evolution of prestin, the motor protein of cochlear outer hair cells. J Neurophysiol 105:36-44. CrossRef Medline

van Netten SM, Kros CJ (2000) Gating energies and forces of the mammalian hair cell transducer channel and related hair bundle mechanics. Proc Biol Sci 267:1915-1923. CrossRef Medline

Vilfan A, Duke T (2003) Two adaptation processes in auditory hair cells together can provide an active amplifier. Biophys J 85:191-203. CrossRef Medline

Wu YC, Ricci AJ, Fettiplace R (1999) Two components of transducer adaptation in auditory hair cells. J Neurophysiol 82:2171-2181. Medline

Zheng J, Shen W, He DZ, Long KB, Madison LD, Dallos P (2000) Prestin is the motor protein of cochlear outer hair cells. Nature 405:149-155. CrossRef Medline 